\title{
The lymphatic system in alcohol-associated liver disease
}

\author{
Reiichiro Kondo ${ }^{1,2}$ and Yasuko Iwakiri' \\ 'Section of Digestive Diseases, Department of Internal Medicine, Yale University School of Medicine, New Haven, CT, USA; ${ }^{2}$ Depart- \\ ment of Pathology, Kurume University School of Medicine, Kurume, Japan
}

The lymphatic system plays vital roles in interstitial fluid balance and immune cell surveillance. The effect of alcohol on the lymphatic system is poorly understood. This review article explores the role of the lymphatic system in the pathogenesis of alcohol-related disease including alcoholic liver disease (ALD) and the therapeutic potential of targeting hepatic lymphatics for the treatment of ALD. (Clin Mol Hepatol 2020;26:633-638)

Keywords: Lymphangiogenesis; Vascular endothelial growth factor C; Inflammation; Lymphatics

\section{INTRODUCTION}

The lymphatic system maintains tissue fluid homeostasis through re-absorption of interstitial fluid. ${ }^{1-7}$ It also regulates inflammatory responses by directing traffic of immune cells via expressing chemokines/chemokine receptors. ${ }^{3-7}$ Alcohol is a frequently abused substance that can permeate nearly all tissues in the body. ${ }^{8}$ Excessive alcohol consumption causes a loss of barrier function of the intestine, leading to translocation of pathogens containing pathogen-associated molecular patterns (PAMPs) to the portal circulation and inducing hepatic inflammation (Fig. 1). ${ }^{9}$

Alcohol remains a major cause of liver disease world-wide. Alcoholic liver disease (ALD) encompasses a spectrum of injury, ranging from simple steatosis to frank cirrhosis. Fatty liver develops in about $90 \%$ of individuals who drink more than $60 \mathrm{~g} /$ day of alcohol. A subset of patients with ALD develops severe alcoholic hepatitis (AH) with a substantially poor prognosis. ${ }^{10}$ Control of in- flammation is an obvious therapeutic focus for the treatment of AH. Hepatic lymphatics could represent therapeutic targets of ALD/AH, because they are highly involved in regulation of inflammatory responses by draining extravasated fluid, antigens, inflammatory mediators, and antigen-presenting cells from the periphery to lymph nodes and are thought to facilitate resolution of tissue inflammation. ${ }^{2-7}$ However, the role of lymphatics in the pathogenesis of alcohol-related disease has not adequately been studied.

In this review article, we first summarize current knowledge of the lymphatic system in health and disease. Second, we introduce some studies that investigated effects of alcohol abuse on the lymphatic system. Third, we address the lymphatic system in the liver in normal and pathological conditions, which includes the potential role of hepatic lymphatics in the pathogenesis of ALD. Fourth, we will conclude with a discussion on the therapeutic potential of hepatic lymphatics for the treatment of ALD.

\begin{abstract}
Abbreviations:
ALD, alcoholic liver disease; LECs, lymphatic endothelial cells; LSECs, liver sinusoidal endothelial cells; PAMPs, pathogen-associated molecular patterns; PBC, primary biliary cholangitis; PD-L1, programmed death-ligand 1; PSC, primary sclerosing cholangitis; VEGF, vascular endothelial growth factor; VEGFR, vascular endothelial growth factor receptor
\end{abstract}

\section{Corresponding author: Yasuko Iwakiri}

Section of Digestive Diseases, Department of Internal Medicine, Yale University School of Medicine, 1080 LMP, 333 Cedar Street, Section of Digestive Diseases, New Haven, CT 06520, USA

Tel: +1-203-785-6204, Fax: +1-203-785-7273

E-mail: yasuko.iwakiri@yale.edu

https://orcid.org/0000-0001-8032-5654 


\section{CLINICAL and MOLECULAR}

\section{HEPATOLOGY}

Volume_26 Number_4 October 2020

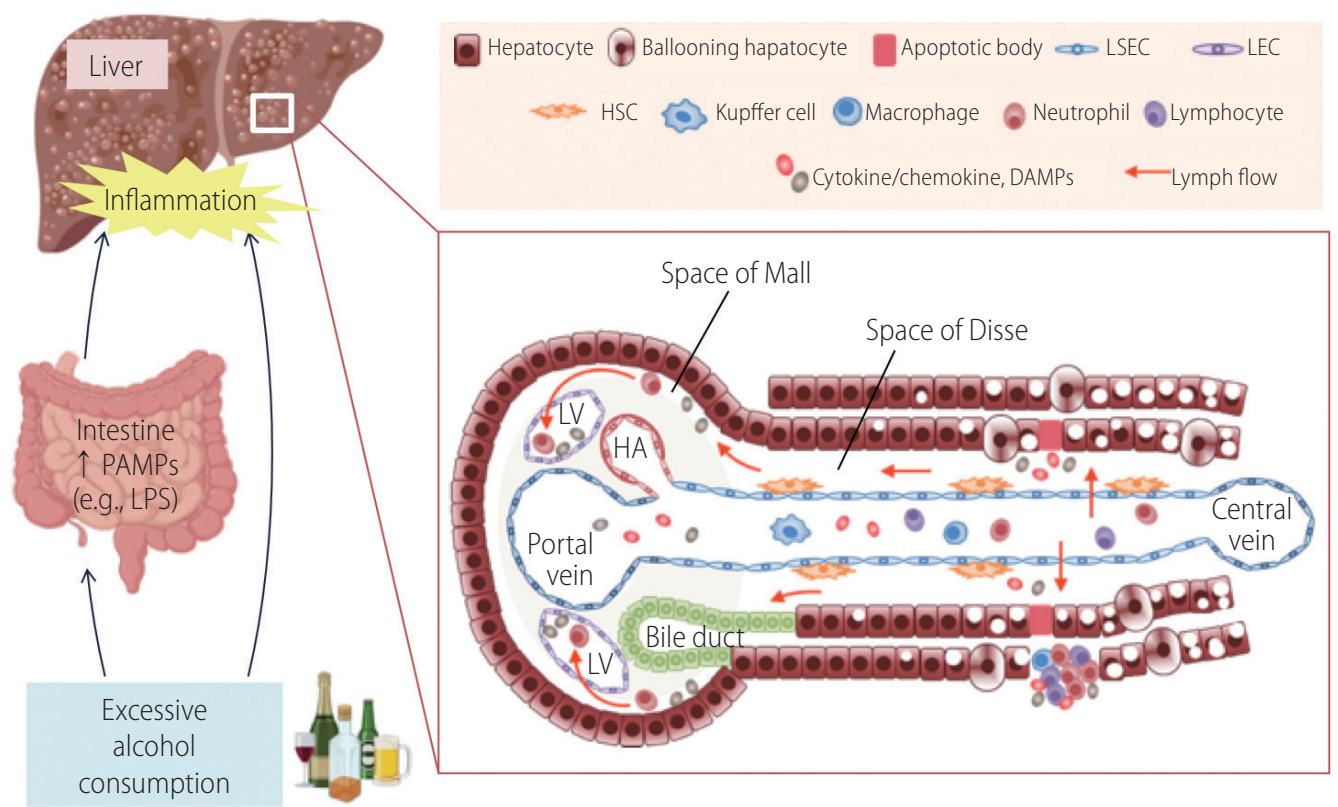

Figure 1. Hepatic lymphatics in alcoholic liver disease. Interstitial fluid in the space of Disse flows through the space of Mall into the interstitium of the portal tract and then into initial lymphatics. Lymphatics are involved in regulation of inflammatory responses by draining interstitial fluid, inflammatory mediators and immune cells from the liver. Excessive alcohol consumption causes a loss of barrier function of the intestine, leading to translocation of PAMPs including microbes and microbial products to the portal circulation. In addition, alcohol and its metabolites induce hepatocyte cell death, resulting in the release of DAMPs in the liver. Both PAMPs and DAMPs activate multiple cell types, including immune cells, hepatocytes, Kupffer cells, HSCS and LSECS, to release inflammatory chemokines and cytokines. Promoting lymphangiogenesis may mitigate inflammation by enhancing discharge of these inflammatory mediators. LSEC, liver sinusoidal endothelial cell; LEC, lymphatic endothelial cell; HSC, hepatic stellate cell; DAMPs, damage-associated molecular patterns; PAMPs, pathogen-associated molecular patterns; LPS, lipopolysaccharide; LV, lymphatic vessel; HA, hepatic artery.

\section{THE LYMPHATIC SYSTEM IN HEALTH AND DIS- EASE}

The lymphatic system has two primary functions, interstitial fluid balance and immune cell surveillance. ${ }^{1-7}$ It maintains interstitial fluid balance by shifting fluid from the periphery to lymph nodes through lymphatic vessels. Interstitial fluid is initially collected by lymphatic capillaries (initial lymphatics) and transported to collecting lymphatics in the form of lymph. ${ }^{1-3,5-7}$ Lymph eventually runs to the two lymph ducts, the right lymph duct and the thoracic duct, where it is returned to the blood circulation via the subclavian veins. ${ }^{3,6,7}$

Lymphatic capillaries are terminal lymphatics with blind-ended structure and serve as a site of fluid exchange, similar to blood capillaries. They are 35-70 $\mu \mathrm{m}$ in diameter and form interconnected networks. Lymphatic capillaries are lined by a single layer of lymphatic endothelial cells (LECS) with lack of a continuous basement membrane and are highly permeable with discontinuous "button-like" junctions through which interstitial fluid, macromolecules and immune cells can be transported. ${ }^{1-3,5-7}$ Lymphatic vessel markers are generally those specific to LECs, including LYVE-1, podoplanin, Prox1, and vascular endothelial growth factor receptor (VEGFR)-3. ${ }^{1,3-7}$ LYVE-1, Prox1, and VEGFR-3 are highly expressed in lymphatic capillaries but low in collecting lymphatics, while expression of podoplanin is persistent throughout the lymphatic network. ${ }^{7}$

In the postnatal stage, lymphatic vessels are usually quiescent, and lymphangiogenesis (formation of new lymphatic vessels from existing vessels) generally occurs in pathologic conditions such as tissue repair, inflammation, and tumor-related conditions, ${ }^{2,3,5-7}$ similar to angiogenesis. Many cytokines and growth factors cooperate in the development and maintenance of lymphatic vessels. The major pro-lymphangiogenic factor is vascular endothelial growth factor (VEGF)-C. Cell signaling via VEGF-C or D and their receptor, VEGFR-3, is the best-studied pathway for lymphangiogenesis. $^{2,3,5-7}$

Lymphatic vessels are heavily involved in regulation of inflammatory responses by discharging extravasated fluid, antigens, inflammatory mediators, and antigen-presenting cells from the periphery to lymph nodes, where immune responses are taking 
place. They also help to clear other types of leukocytes from sites of inflammation. Further, LECs of lymphatic vessels attract activated dendritic cells, T cells, and B cells that express CCR7 and CCR10 by secreting the ligands for these receptors, CCL21 and CCL27..$^{3-7}$ In addition, LECs express a chemokine receptor D6, which internalizes and degrades inflammatory beta-chemokines, thereby reducing recruitment of monocytes and $T$ cells to inflamed tissues." Furthermore, LECs have been shown to secrete immunosuppressive agents such as transforming growth factor- $\beta 1 .^{12} \mathrm{LECS}$ in lymph nodes also exhibit high levels of programmed death-ligand 1 (PD-L1), a T cell inhibitory receptor ligand, and lack costimulatory signals, thus attenuating $T$ cell activation and inducing peripheral tolerance. ${ }^{13}$

Lymphangiogenesis is thought to be a beneficial event for tissue repair and clearing inflammation. It can increase collection of interstitial fluid and migration of inflammatory cells out of affected tissues, interfere with recruitment of inflammatory cells, and enhance clearance of antigens and proinflammatory mediators, leading to mitigation of inflammation. ${ }^{14-18}$

\section{ALCOHOL USE DISORDER AND THE LYMPHAT- IC SYSTEM}

There are only a small number of studies that have investigated the effect of alcohol on the functions of the lymphatic system. However, their results strongly support a role for the lymphatic system in the pathogenesis of ALD. Studies using rats have shown a significantly increased mesenteric lymph flow when ethanol diets were administered intragastrically as a bolus or as a continuous infusion into the duodenum. ${ }^{19,20}$ Alcohol can reduce the barrier function of vascular endothelial cells, which increases permeability of the mesenteric microcirculation. This could lead to fluid accumulation in interstitial spaces of mesenteric tissues and increased drainage to lymphatics. It has also been reported that alcohol disrupts a tight junction protein, VE-cadherin, in human umbilical vein endothelial cells and that alcohol feeding significantly increases FITC-albumin extravasation from the mesenteric microcirculation in rats. ${ }^{21}$

Other studies using rats have shown that acute alcohol intoxication and chronic alcohol administration increase mesenteric lymphatic permeability and lymphatic leakage to perilymphatic tissues. $^{22,23}$ Lymphatic leakage to the perilymphatic adipose tissue causes local inflammation, as indicated by elevated proinflammatory cytokine expression and neutrophil density in this tissue. ${ }^{23}$
Generally, it is considered that lymphatic drainage from sites of inflammation reduces inflammation. Under excessive alcohol exposure, however, the drainage function of lymphatics may become inadequate, and perilymphatic tissue leakage seems to result. Preventing lymphatic leakage could be a therapeutic strategy for alcohol-induced tissue inflammation.

Studies using human subjects have reported that alcohol consumption increases thoracic duct lymph flow, ${ }^{24}$ and that thoracic duct diameters of patients with alcoholic cirrhosis are greater than those of patients with nonalcoholic cirrhosis. ${ }^{25}$ It is unclear how alcohol could further increase thoracic duct lymph flow in cirrhotic patients, but increased permeability of blood capillaries and increased drainage of interstitial fluid to lymphatics may account for the mechanism as observed in ethanol-fed rats. ${ }^{19-21}$ Another possibility may include alcohol's effect on increased production of lymph in cirrhotic livers by influencing hepatic sinusoidal vasculature and/or its effect on increased hepatic lymphangiogenesis to accommodate more lymphatic fluid produced in the liver. All these observations strongly indicate the potential role of the lymphatic system in alcohol-related disease including ALD.

\section{THE HEPATIC LYMPHATIC SYSTEM IN DISEASE}

Hepatic lymphatic fluid is thought to be produced from plasma components transferred through the fenestrae of liver sinusoidal endothelial cells (LSECs) into the space of Disse, an interstitial space between LSECS and hepatocytes (Fig. 1; for structural details and functions, please also refer to references 3 and 4). The interstitial fluid in the space of Disse primarily (roughly $80 \%$ ) flows through the space of Mall, a space between the stroma of the portal tract and the outermost hepatocytes, into the interstitium of the portal tract and then into initial lymphatics. ${ }^{1-4}$ Some portion of the interstitial fluid in the space of Disse flows into the interstitium around the central vein, which is located in the center of the liver lobules and connected to the hepatic vein, or underneath the hepatic capsule. ${ }^{3,26}$ Initial lymphatics in the portal tract merge into collecting lymphatics and drain to lymph nodes at the hepatic hilum, while lymphatic vessels along the central vein run along the inferior vena cava through the diaphragm toward posterior mediastinal lymph nodes. Lymphatic fluid running underneath the capsule of the convex surface of the liver drains to mediastinal lymph nodes through the coronary ligament, while lymphatic fluid running along the concave surface of the liver drains to lymph nodes in the hepatic hilum and to regional lymph 
nodes. ${ }^{3}$

The hepatic vasculature and hepatic blood circulation are heavily distorted in liver cirrhosis. Similarly, the architecture of hepatic lymphatic vessels and hepatic lymph flow are changed in cirrhosis. In cirrhotic livers, resistance to sinusoidal blood flow increases because of structural deformations of tissues around the sinusoids, portal, and central veins. Consequently, sinusoidal hydrostatic pressure is elevated, which increases the amount of plasma components filtered through the sinusoids into the space of Disse. In patients with liver cirrhosis, lymphatic fluid produced in the liver increases up to 30 -fold. ${ }^{3,4}$

It is reported that protein contents of hepatic lymph are much lower in patients with ALD-associated cirrhosis than in controls. ${ }^{27}$ While lymph within hepatic lymphatics generally has the same protein concentrations as interstitial fluid in the space of Disse, increased plasma components transferred through the sinusoids into the space of Disse in cirrhotic livers can subsequently decrease protein concentrations of interstitial fluid and oncotic pressure in the space of Disse. Consequently, the loss of osmoregulation in cirrhotic livers leads to ballooning of hepatocytes, which is a typical histological feature of $\mathrm{AH}$ and results from accumulation of water, fat and proteins that are normally exported from hepatocytes and deterioration of cytokeratins. ${ }^{28}$ Chronic alcohol consumption may worsen osmoregulation indirectly by altering morphology and function of LSECs. Livers of alcohol-fed rats are characterized by massive loss of fenestrae of $\mathrm{LSECs}^{29}$ and dysfunctional LSECs characterized by a decreased production of nitric oxide, ${ }^{30}$ leading to increased resistance to sinusoidal blood flow and increased sinusoidal hydrostatic pressure.

Lymphatics play a role in immune cell recruitment and trafficking during chronic liver disease. LECs secrete CCL21 and attract activated T cells and B cells expressing its receptor CCR7..$^{3-7} \mathrm{~A}$ study showed an increased number of CCL21-expressing lymphatics in the livers of patients with nonalcoholic steatohepatitis. ${ }^{31}$ Other chronic inflammatory liver diseases including primary biliary cholangitis (PBC), primary sclerosing cholangitis (PSC), and ALD also exhibit increased expression of CCL21 in the liver, but the relationship between these increases of CCL21 and hepatic lymphatics has not been investigated. In addition, the increased level of CCL21 expression is much smaller in ALD than in PBC or PSC, ${ }^{32}$ suggesting that the magnitude of CCL21 involvement is etiologyspecific. It would be interesting to know whether alcohol influences CCL21 expression in hepatic LECs.

\section{LYMPHATIC-MODULATION AS POTENTIAL THERAPY}

Delivery of lymphangiogenic factors, such as VEGF-C, to targeted organs has been shown to reduce inflammation in a variety of disease models. ${ }^{6,14-18}$ For example, virus-mediated delivery of VEGF-C alleviated chronic skin inflammation, ${ }^{15}$ inflammatory bowel disease ${ }_{1}^{16}$ tubulointerstitial nephritis ${ }^{17}$ and hepatic encephalopathy $y^{33}$ in experimental models using mice or rats. In a mouse model of psoriasis-like skin inflammation, targeted delivery of VEGF-C to sites of inflammation led to a significant increase of lymphatic vessels in inflamed skin and improved lymphatic drainage function. At the same time, this treatment significantly reduced infiltration of inflammatory cells in inflamed skin. ${ }^{18}$ These studies suggest that promoting lymphangiogenesis may be a novel potential therapeutic strategy, particularly for diseases where inflammation plays a key role in their pathogenesis.

LECs/lymphatics also express a chemokine receptor D6, which helps to alleviate inflammation. ${ }^{11,34}$ The D6 receptor can actively internalize and degrade inflammatory beta-chemokines (a.k.a., CC chemokines), ${ }^{11}$ such as CCL2 (a.k.a., monocyte chemoattractant protein-1; MCP-1) and CCL5 (a.k.a., RANTES), resulting in resolution of tissue inflammation. ${ }^{11}$ CCL2 induces recruitment of monocytes from blood to tissues to become macrophages, while CCL5 attracts T-cells, eosinophils, and basophils that express a receptor CCR5. It is known that circulating CCL2 and CCL5 as well as macrophages and T-cells are upregulated in patients with ALD. ${ }^{35}$ Because the progression of ALD is largely driven by inflammation, facilitating lymphangiogenesis may be a potential treatment of ALD via increasing D6 levels as well as enhancing lymphatic drainage of pro-inflammatory molecules.

\section{CONCLUSIONS AND PERSPECTIVES}

Only a few studies have investigated how alcohol influences LEC phenotype and function, as well as the effects of these changes on disease pathogenesis. In particular, it is largely unknown whether hepatic lymphatics are involved in the pathogenesis of ALD. Given that the lymphatic system plays an essential role in immune cell surveillance and that inflammation is a key contributor to the development of ALD, it is likely that lymphatics play an important role in the pathogenesis of ALD. Therefore, studies on the lymphatic system in relation to ALD are likely to yield significant advances in our understanding of the disease. 
Targeted delivery of VEGF-C and tissue-specific induction of lymphangiogenesis represent a potentially new and promising approach for the treatment of chronic inflammatory diseases like ALD.

\section{Authors' contribution}

$\mathrm{YI}$ and RK contributed to review concept and design; drafting of the manuscript; and critical revision of the manuscript for important intellectual content.

\section{Acknowledgements}

NIH grants (R01AA025342, R01DK117597 and R56DK121511)

to YI. We would like to thank Drs. Teruo Utsumi and Matthew

McConnell for their critical comments and edits.

\section{Conflicts of Interest}

The authors have no conflicts to disclose.

\section{REFERENCES}

1. Chung C, Iwakiri Y. The lymphatic vascular system in liver diseases: its role in ascites formation. Clin Mol Hepatol 2013;19:99-104.

2. Iwakiri Y. The Lymphatic system: a new frontier in hepatology. Hepatology 2016;64:706-707.

3. Tanaka M, Iwakiri Y. the hepatic lymphatic vascular system: structure, function, markers, and lymphangiogenesis. Cell Mol Gastroenterol Hepatol 2016;2:733-749.

4. Tanaka M, Iwakiri Y. Lymphatics in the liver. Curr Opin Immunol 2018;53:137-142.

5. Alitalo K. The lymphatic vasculature in disease. Nat Med 2011;17: 1371-1380.

6. Bouta EM, Bell RD, Rahimi H, Xing L, Wood RW, Bingham CO 3rd, et al. Targeting lymphatic function as a novel therapeutic intervention for rheumatoid arthritis. Nat Rev Rheumatol 2018;14:94-106.

7. Breslin JW, Yang Y, Scallan JP, Sweat RS, Adderley SP, Murfee WL. Lymphatic vessel network structure and physiology. Compr Physiol 2018:9:207-299.

8. Souza-Smith FM, Lang CH, Nagy LE, Bailey SM, Parsons LH, Murray GJ. Physiological processes underlying organ injury in alcohol abuse. Am J Physiol Endocrinol Metab 2016;311:E605-E619.

9. Gao B, Ahmad MF, Nagy LE, Tsukamoto H. Inflammatory pathways in alcoholic steatohepatitis. J Hepatol 2019;70:249-259.

10. Crabb DW, Im GY, Szabo G, Mellinger JL, Lucey MR. Diagnosis and treatment of alcohol-associated liver diseases: 2019 practice guidance from the American Association for the Study of Liver Diseases.
Hepatology 2020;71:306-333.

11. Jamieson T, Cook DN, Nibbs RJ, Rot A, Nixon C, McLean P, et al. The chemokine receptor D6 limits the inflammatory response in vivo. Nat Immunol 2005;6:403-411.

12. Christiansen AJ, Dieterich LC, Ohs I, Bachmann SB, Bianchi R, Proulx ST, et al. Lymphatic endothelial cells attenuate inflammation via suppression of dendritic cell maturation. Oncotarget 2016;7:3942139435.

13. Tewalt EF, Cohen JN, Rouhani SJ, Guidi CJ, Qiao H, Fahl SP, et al. Lymphatic endothelial cells induce tolerance via PD-L1 and lack of costimulation leading to high-level PD-1 expression on CD8 T cells. Blood 2012;120:4772-4782.

14. Zhou Q, Guo R, Wood R, Boyce BF, Liang Q, Wang YJ, et al. Vascular endothelial growth factor $\mathrm{C}$ attenuates joint damage in chronic inflammatory arthritis by accelerating local lymphatic drainage in mice. Arthritis Rheum 2011;63:2318-2328.

15. Huggenberger R, Ullmann S, Proulx ST, Pytowski B, Alitalo K, Detmar M. Stimulation of lymphangiogenesis via VEGFR-3 inhibits chronic skin inflammation. J Exp Med 2010;207:2255-2269.

16. D'Alessio S, Correale C, Tacconi C, Gandelli A, Pietrogrande G, Vetrano $S$, et al. VEGF-C-dependent stimulation of lymphatic function ameliorates experimental inflammatory bowel disease. J Clin Invest 2014;124:3863-3878.

17. Hasegawa S, Nakano T, Torisu K, Tsuchimoto A, Eriguchi M, Haruyama $\mathrm{N}$, et al. Vascular endothelial growth factor- $\mathrm{C}$ ameliorates renal interstitial fibrosis through lymphangiogenesis in mouse unilateral ureteral obstruction. Lab Invest 2017;97:1439-1452.

18. Schwager S, Renner S, Hemmerle T, Karaman S, Proulx ST, Fetz R, et al. Antibody-mediated delivery of VEGF-C potently reduces chronic skin inflammation. JCI Insight 2018;3:e124850.

19. Baraona $E$, Lieber CS. Intestinal lymph formation and fat absorption: stimulation by acute ethanol administration and inhibition by chronic ethanol administration and inhibition by chronic ethanol feeding. Gastroenterology 1975;68:495-502.

20. Hayashi H, Nakata K, Mótohashi Y, Takano T. Acute inhibition of lipid transport in rat intestinal lymph by ethanol administration. Alcohol Alcohol 1992;27:627-632.

21. Doggett TM, Breslin JW. Acute alcohol intoxication-induced microvascular leakage. Alcohol Clin Exp Res 2014;38:2414-2426.

22. Souza-Smith FM, Simon L, Siggins R, Molina PE. Alcohol-induced mesenteric lymphatic permeability: link to immunometabolic modulation of perilymphatic adipose tissue. Int J Mol Sci 2019;20:4097.

23. Souza-Smith FM, Siggins RW, Molina PE. mesenteric lymphatic-perilymphatic adipose crosstalk: role in alcohol-induced perilymphatic adipose tissue inflammation. Alcohol Clin Exp Res 2015;39:13801387.

24. Bartos V, Brzek V. Effect of acute ethanol administration on the thoracic duct lymph flow in man. Lymphology 1978;11:54-56. 


\section{CLINICAL and MOLECULAR
HEPATOLOGY}

25. Takahashi H, Kuboyama S, Abe H, Aoki T, Miyazaki M, Nakata H. Clinical feasibility of noncontrast-enhanced magnetic resonance lymphography of the thoracic duct. Chest 2003;124:2136-2142.

26. Kitazume N. Studies on normal architecture of subserosal lymph vessels of the human liver. Kanzo 1983;24:581-590.

27. Witte $\mathrm{MH}$, Witte $\mathrm{CL}$, Dumont AE. Estimated net transcapillary water and protein flux in the liver and intestine of patients with portal hypertension from hepatic cirrhosis. Gastroenterology 1981;80:265272.

28. Crawford JM. Histologic findings in alcoholic liver disease. Clin Liver Dis 2012;16:699-716.

29. Vollmar B, Menger MD. The hepatic microcirculation: mechanistic contributions and therapeutic targets in liver injury and repair. Physiol Rev 2009;89:1269-1339.

30. Iwakiri Y, Kim MY. Nitric oxide in liver diseases. Trends Pharmacol Sci 2015;36:524-536.

31. Tamburini BAJ, Finlon JM, Gillen AE, Kriss MS, Riemondy KA, Fu $R$, et al. Chronic liver disease in humans causes expansion and differentiation of liver lymphatic endothelial cells. Front Immunol
2019;10:1036.

32. Grant AJ, Goddard S, Ahmed-Choudhury J, Reynolds G, Jackson DG, Briskin $M$, et al. Hepatic expression of secondary lymphoid chemokine (CCL21) promotes the development of portal-associated lymphoid tissue in chronic inflammatory liver disease. Am J Pathol 2002;160:1445-1455.

33. Hsu SJ, Zhang C, Jeong J, Lee SI, McConnell M, Utsumi T, et al. Enhanced meningeal lymphatic drainage ameliorates neuroinflammation and hepatic encephalopathy in cirrhotic rats. BioRxiv. 2020 May 3. doi: 10.1101/2020.05.01.072611.

34. Nibbs RJ, Kriehuber E, Ponath PD, Parent D, Qin S, Campbell JD, et al. The $\beta$-chemokine receptor D6 is expressed by lymphatic endothelium and a subset of vascular tumors. Am J Pathol 2001;158:867877.

35. Ambade A, Lowe P, Kodys K, Catalano D, Gyongyosi B, Cho Y, et al. Pharmacological inhibition of CCR2/5 signaling prevents and reverses alcohol-induced liver damage, steatosis, and inflammation in mice. Hepatology 2019;69:1105-1121. 